\title{
Mucosal immunization with the Moraxella catarrhalis porin M35 induces enhanced bacterial clearance from the lung: a possible role for opsonophagocytosis
}

\author{
Donna M. Easton ${ }^{1,2,3}$, Allan W. Cripps ${ }^{4}{ }^{*}$, A. Ruth Foxwell ${ }^{1}$ and Jennelle M. Kyd \\ ${ }^{1}$ Faculty of Health, University of Canberra, Canberra, ACT, Australia \\ 2 School of Veterinary Science, University of Queensland, Gatton, QLD, Australia \\ ${ }^{3}$ Australian Infectious Disease Research Centre, School of Chemistry and Molecular Biosciences, University of Queensland, St Lucia, QLD, Australia \\ ${ }^{4}$ School of Medical Science, Griffith Health Institute, Griffith University, Gold Coast campus, Gold Coast, OLD, Australia \\ ${ }^{5}$ Capricornia Centre for Mucosal Immunology, Central Queensland University Australia, Rockhampton, QLD, Australia
}

\section{Edited by:}

Eric Cox, Ghent University, Belgium

Reviewed by:

Diane Bimczok, University of Alabama

at Birmingham, USA

Jean-Pierre Yves Scheerlinck, The

University of Melbourne, Australia

*Correspondence:

Allan W. Cripps, School of Medical Science, Griffith Health Institute, Griffith University, Gold Coast Campus, Gold Coast, QLD, Australia e-mail:allan.cripps@griffith.edu.au
Moraxella catarrhalis is a significant cause of respiratory tract infection against which a vaccine is sought. Several outer membrane proteins are currently under investigation as potential vaccine antigens, including the porin M35. We have previously shown that the third external loop of M35 was immunodominant over the remainder of the protein for antibody produced in mice against the refolded recombinant protein. However, as this loop is predicted to fold inside the porin channel we also predicted that it would not be accessible to these antibodies when M35 is expressed on the surface of the bacteria in its native conformation. This study investigated the functional activity of antibodies against M35 and those specific for the loop 3 region of M35 in vitro and in vivo. Antisera from mice immunized with M35 or the loop 3-deletion, M35loop3- ${ }^{-}$, recombinant proteins were not bactericidal but did have enhanced opsonic activity, whereas antibodies raised against the loop 3 peptide were not opsoniszing indicating that the immunodominant loop 3 of M35 was not accessible to antibody as we had previously predicted. Mucosal immunization with M35, M35 that had an antigenically altered loop 3 [M35(ID78)] and M35loop3enhanced the clearance of $M$. catarrhalis from the lungs of mice challenged with live $M$. catarrhalis. The in vivo clearance of bacteria in the mice with the M35-derived protein constructs correlated significantly $(p<0.001)$ with the opsonic activity assessed an in vitro opsonophagocytosis assay. This study has demonstrated that the immunodominant B-cell epitope to loop 3 of the M. catarrhalis outer membrane protein M35 is not associated with immune protection and that M35-specific antibodies are not bactericidal but are opsoniszing. The opsoniszing activity correlated with in vivo clearance of the bacteria suggesting that opsoniszing antibody may be a good correlate of immune protection.

Keywords: Moraxella catarrhalis, porin, mucosal immunization, opsonophagocytosis, respiratory tract

\section{INTRODUCTION}

Moraxella catarrhalis is a Gram-negative bacterium that is often the causual organism for respiratory tract infections such as otitis media, sinusitis, and exacerbations of chronic obstructive pulmonary disease (COPD; Murphy, 1996; Karalus and Campagnari, 2000; Verduin et al., 2002). On rare occasions this bacterium can cause more serious diseases such as meningitis and septicaemia (Meyer et al., 1995; Daoud et al., 1996). Additionally, the presence of $M$. catarrhalis appears to influence the pathogenicity of other respiratory pathogens, such as Haemophilus influenzae (Armbruster et al., 2010) and Streptococcus pneumoniae (Krishnamurthy et al., 2009). A vaccine against M. catarrhalis is sought and a number of outer membrane proteins are currently being characterized and evaluated for their potential as vaccine antigens (Murphy, 2005, 2009; Massa et al., 2009; Mawas et al., 2009). We have previously reported the characterization of an OMP from M. catarrhalis, designated M35, that we are investigating for its functional role and potential as a vaccine antigen.

Our initial characterization of M35 suggested that it possesses the desired characteristics of a vaccine antigen in that it is highly conserved, surface exposed, and immunogenic in an animal model (Easton et al., 2005). Subsequent studies confirmed a high level of conservation of M35 across strains, as well as demonstrating an important role for M35 in resistance to aminopenicillins (Jetter et al., 2009) and involvement in several stress responses. M35 shares homology with the family of outer membrane porins and is likely to conform to the usual tertiary structure of porins in which the third of eight external loops folds into the lumen of a channel formed by 16 anti-parallel $\beta$-sheets (Seshadri et al., 1998). In the predicted structure of M35 the third external loop is one of three loops that are much longer than the other loops (Figure 1). M35 contains an immunodominant epitope in the third external loop 
and we hypothesized that if loop 3 folded into the porin channel then it would not be accessible to antibody (Easton et al., 2005). By directing the majority of the antibody response against an inaccessible epitope, the bacteria could protect this protein from the host's immune system and subsequent immunological selective pressure. This could potentially explain why M35 is so highly conserved, while other similar OMPs tend to have variable surface regions in response to selective pressure from the host's immune system (Haase et al., 1991; Bash et al., 1995; Schirmer, 1998; Mes and van Putten, 2007).

M35 was also shown to have epitopes that are accessible to antibody binding on the surface of the bacterium (Easton et al., 2005). If the above hypothesis is correct, these surface epitopes would not include loop 3. As part of identifying the specificity of antibody to loop 3 it was also found that a single amino acid substitution in loop 3 (in the isolate ID78LN266) was sufficient to significantly reduce recognition of $\mathrm{M} 35$ by antibody. In this context, both the conformational significance of the loop and single point mutations within immunodominant loop regions have been identified as affecting antibody binding for the P2 porin of non-typeable Haemophilus influenzae (NTHi; Duim et al., 1993; Yi and Murphy, 1997). The lack of M35-specific antibody binding to the surface of the variant isolate implied that the majority of antibodies raised in mice against M35 were specific for loop 3 (where the mutation is located) and that loop 3 might be surface exposed. Such a result would suggest that either the predicted folding of M35 was incorrect or that loop 3 is either partially or fully outside the channel in M35, which would be highly unusual for this type of an outer membrane porin.

The aims of this study were threefold. Firstly, to further investigate the surface accessibility of antibodies directed against M35, specifically the loop 3 region. Secondly, to determine the bactericidal and opsonizing activity of M35-specific and loop 3-specific antibodies in vitro. Thirdly, to determine the efficacy of M35 as a protective antigen in a mouse mucosal immunization pulmonary clearance model (Kyd et al., 1998, 1999; Murphy et al., 1998).

\section{MATERIALS AND METHODS \\ CLONING AND EXPRESSION OF M35-DERIVED PROTEIN CONSTRUCTS}

Three recombinant proteins based on M35 were constructed: (1) M35(ID78), the full M35 protein based on the gene from the

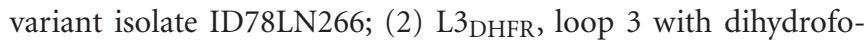
late reductase (DHFR) as a carrier protein; and (3) DHFR alone as a control. M35(ID78) contains two amino acid substitutions in comparison to the consensus M35 sequence, one of which was found previously to be antigenically significant (Easton et al., 2005). In addition to these newly constructed recombinant proteins, the consensus M35, the mature M35 (M35mat), and M35 without the loop 3 region (M35loop $3^{-}$) were also used in this study (Easton et al., 2005). The relative structures of the recombinant proteins used in this study are represented diagrammatically in Figure 1. All recombinant proteins expressed a $6 \times$ His tag sequence at the $\mathrm{N}$-terminal and were produced by expression in M15 [pRep4] Escherichia coli (Qiagen) with purification by nickel-nitrilotriacetic acid column chromatography according the manufacturer's protocol for this expression system under denaturing conditions ( $8 \mathrm{M}$ urea). M35 and the variant forms were

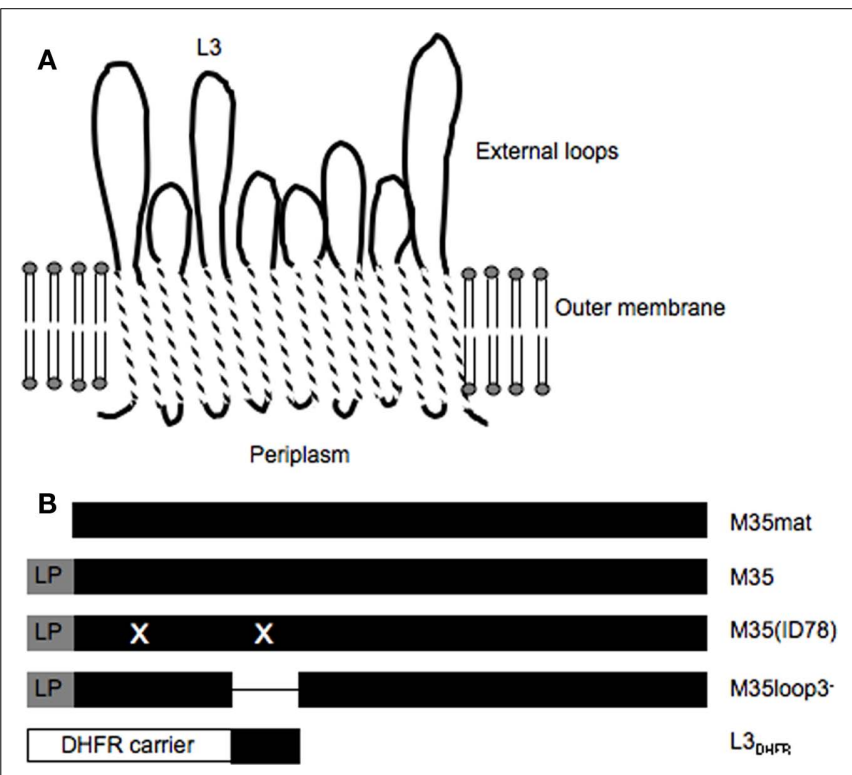

FIGURE 1 | Diagramatic representation of the structure of M35 and the M35-derived recombinant proteins. (A) Schematic of M35 showing the relative lengths of the external loops. (B) Relative sequence inclusions of the recombinant protein constructs. LP, leader peptide and $X$, amino acid substitution. M35mat does not include the leader peptide; M35 is the full sequence including the leader peptide; M35(ID78) includes the two amino acid substitutions present in M35 from isolate ID78LN266 when compared to the consensus, which are valine to isoleucine at position 32 and glycine to aspartic acid at position 133; M35loop3- ${ }^{-}$does not include the loop 3 region; $L 3_{\text {DHFR }}$ is the loop 3 region of M35 attached to the DHFR carrier.

refolded as previously described (Watanabe, 2002; Easton et al., 2005).

M35(ID78) was cloned by amplifying the M35 gene sequence from genomic DNA extracted from the ID78LN266 isolate of $M$. catarrhalis, as described previously for the consensus M35 using the pQE30 vector (Qiagen) (Easton et al., 2005).

L3 3 DHFR was produced by amplifying the gene sequence coding for the loop 3 region of the M35 gene from genomic DNA extracted from the 4223 isolate of $M$. catarrhalis using primers DEM35L3R (antisense) GCCCTGCAGATTGTTGGCACG and DEM35L3F (sense) GCCAGATCTATTGATGACAGTGTTG. These primers introduced SphI and Pst I restriction enzyme recognition sites at either end of the PCR product that enabled directional insertion into the vector. The pQE40 vector (Qiagen) was used because this vector contains the coding sequence for DHFR immediately preceding the multiple cloning site and the recombinant protein produced therefore contains the carrier at the $\mathrm{N}$-terminal end of the construct.

\section{PRODUCTION OF HYPERIMMUNE MOUSE SERUM}

Antisera was raised for each of the M35-derived proteins and whole killed M. catarrhalis in male BALB/c mice. Mice were immunized intraperitoneally three times at weekly intervals with $10 \mu \mathrm{g}$ of each of the M35-derived protein constructs or $10^{8}$ whole killed $M$. catarrhalis emulsified with an equal volume of incomplete Freund's adjuvant (IFA). The antibody concentration (total IgG) was measured by enzyme linked immunosorbent assay (ELISA) 
against the specific antigen. The immunization experiments were approved by the University of Canberra Committee for Ethics in Animal Experimentation and the Central Queensland University Animal Ethics Committee.

\section{ENZYME LINKED IMMUNOSORBENT ASSAY}

Enzyme linked immunosorbent assay was used to measure specific IgG and IgA as described previously (Kyd et al., 1999), however the volumes were reduced to $50 \mu \mathrm{L}$ and the assay was developed using Zymed ${ }^{\circledR}$ 3,3',5,5'-tetramethylbenzidine (TMB) single solution (Invitrogen). The ELISA plates were coated with $0.5 \mu \mathrm{g}$ of the relevant antigen for each group. The whole killed 4223 cells were sonicated prior to coating for the WKC group. The serum and bronchoalveolar lavage (BAL) were serially diluted starting at $1 / 5$ and $1 / 2$, respectively. The antibody concentration was measured for each mouse individually against the same antigen with which the mouse was immunized and the samples from the non-immunized mice were tested for antibody against both M35 and the whole $M$. catarrhalis cells. The limit of detection was approximately $0.2 \mu \mathrm{g} / \mathrm{mL}$ for IgG and $0.125 \mu \mathrm{g} / \mathrm{mL}$ for IgA.

\section{FLOW CYTOMETRY}

Binding of antibodies raised against the M35-derived protein constructs to the surface of $M$. catarrhalis cells was analyzed by flow cytometry. M. catarrhalis isolates 4223 and ID78LN266 were grown overnight on brain heart infusion agar (Oxoid) supplemented with 5\% defibrinated horse blood, washed in sterile PBS with $0.5 \%$ BSA and adjusted to a concentration of $1 \times 10^{10} \mathrm{CFU} / \mathrm{mL}$ using the optical density (OD) at $400 \mathrm{~nm}$. One hundred microliter aliquots of the cell suspensions were centrifuged and resuspended in the mouse anti-serum diluted to approximately $20 \mu \mathrm{g} / \mathrm{mL}$ with sterile PBS with $0.5 \% \mathrm{BSA}$ and incubated for $1 \mathrm{~h}$ at $37^{\circ} \mathrm{C}$ to allow the antibodies to bind. The bacteria were centrifuged and resuspended in a 1/200 dilution of goat antimouse IgG AlexaFluor488 (Molecular Probes, Invitrogen) and incubated for $1 \mathrm{~h}$ at $37^{\circ} \mathrm{C}$. The labeled cells were washed twice in sterile PBS before analysis with a flow cytometer (Coulter XLMCL, Beckman Coulter). About 100,000 events were counted and binding of antibodies was assessed by observation of the curve shift in comparison to bacteria exposed to serum from non-immunized mice.

\section{EVALUATION OF COMPLEMENT SENSITIVITY}

The isolate 4223 was not used for the bactericidal and opsonophagocytosis assays because, like many other M. catarrhalis isolates, it is complement resistant. Five isolates from our collection that had originally been collected by nasopharyngeal swab from healthy children with asymptomatic carriage were tested because these isolates are more likely to be serum sensitive than isolates taken from infection ( $\mathrm{Hol}$ et al., 1995). To assess the complement sensitivity of these isolates the culture and spot test described by Verduin et al. (1995) was used. The isolates were grown overnight with shaking at $37^{\circ} \mathrm{C}$ in Mueller-Hinton broth (Oxoid). The bacteria were pelleted by centrifugation and resuspended in veronal buffered saline $\left(\mathrm{VBS}^{2+} ; 5 \mathrm{mM}\right.$ barbitone, $145 \mathrm{mM} \mathrm{NaCl}, 0.15 \mathrm{mM} \mathrm{CaCl}_{2}, 0.5 \mathrm{mM} \mathrm{MgCl}_{2}, \mathrm{pH} 7.4$ ). The concentration was adjusted to approximately $1.5 \times 10^{8} \mathrm{CFU} / \mathrm{mL}$ using the OD at $400 \mathrm{~nm}$. One hundred microliter of this suspension was then spread on 5\% sheep agar plates (Oxoid) and allowed to absorb. Fifty microliter of guinea pig serum, both active and heatinactivated $\left(30 \mathrm{~min}\right.$ at $\left.56^{\circ} \mathrm{C}\right)$, was spotted in duplicate on to each plate and the plates were immediately incubated at $37^{\circ} \mathrm{C}$. After overnight incubation the bacterial growth within the spots was evaluated. This assay was repeated twice and the 2:03KAL isolate was chosen because of consistency in the active serum inhibiting growth and the heat-inactivated serum not inhibiting its growth to any detectable degree. M35 is very well conserved across diverse isolates (Easton et al., 2005), however as this isolate had not been included in our previous study, the expression of M35 and its antigenic conservation with 4223 was confirmed by western blot.

\section{BACTERICIDAL ASSAY}

The bactericidal activity of antibodies raised against the M35derived proteins was assessed using the method described by Murphy et al. (1998). Serum samples from intraperitoneally immunized mice were heat-inactivated by incubation at $56^{\circ} \mathrm{C}$ for $30 \mathrm{~min}$ before use. The serum was also diluted with PBS to give an equivalent concentration of antibody as determined by ELISA, which was necessary because the purpose of the experiment was to compare the bactericidal activity of the antibodies raised against the different proteins rather than the efficacy of immunization with these proteins. The final antibody concentration of each sample was approximately $20 \mu \mathrm{g} / \mathrm{mL}$.

Moraxella catarrhalis isolate 2:03KAL was grown in brain heart infusion (BHI) broth at $37^{\circ} \mathrm{C}$ with shaking at $220 \mathrm{rpm}$ until the $\mathrm{OD}$ at $600 \mathrm{~nm}$ was approximately 0.2 , indicative of early logphase growth. The early log-phase broth culture was diluted 1/4000 with sterile PCGM saline $\left(3.26 \mathrm{mM} \mathrm{NaH}_{2} \mathrm{PO}_{4}, 12.8 \mathrm{mM}\right.$ $\mathrm{NaHPO}_{4}, 125 \mathrm{mM} \mathrm{NaCl}, 1.25 \mathrm{mM} \mathrm{CaCl}_{2}, 0.5 \mathrm{mM} \mathrm{MgCl}_{2}, 1 \%$ gelatin). Twenty microliter of this cell suspension was added to each well of the microtiter plate with $10 \mu \mathrm{L}$ of sterile PBS. Ten microliter of heat-inactivated serum sample was added to each well, with each serum sample being tested in triplicate. Serum from mice immunized with whole formalin-killed $M$. catarrhalis 4223 cells (WKC) was included as a positive control. Control wells without serum received sterile PBS instead of serum. The samples were mixed gently by pipetting and incubated for $30 \mathrm{~min}$ at room temperature to allow the antibodies present in the mouse serum to bind to the bacteria. Ten microliter of guinea pig serum was then added to each well as the complement source and the cells were incubated for a further $30 \mathrm{~min}$ at $37^{\circ} \mathrm{C}$ to allow complement mediated killing of the bacteria. No complement control wells received sterile PBS instead of the guinea pig serum. Two hundred microliter of sterile PCGM saline was added to each well and $50 \mu \mathrm{L}$ aliquots were spotted on to CBA plates in duplicate to enable quantification of live bacteria. The plates were incubated overnight and the number of colony forming units in each spot was counted.

The bacterial counts were compared with the negative control wells that did not have mouse serum, complement source or both. Serum from non-immunized mice was also included as a negative control. A viability control was included that was titrated at the beginning of the incubation period so that survival during the incubation time could be assessed. A positive result is considered 
killing that reduces recovery to $\leq 50 \%$ of the recovery from the negative control (Murphy et al., 1998).

\section{OPSONOPHAGOCYTOSIS ASSAY}

An assay to measure the ability of antibodies raised against the M35-derived proteins to opsonize $M$. catarrhalis and thereby promote phagocytosis of the bacteria was developed with reference to similar assays used to analyze opsonization of other species of bacteria (Aase et al., 1995; Delvig et al., 1997a; Martinez et al., 1999; Jomaa et al., 2005a). M. catarrhalis 2:03KAL was grown overnight on CBA plates, harvested, washed twice in sterile PBS and fluorescently dyed with 5-chloromethylfluorescein diacetate (CMFDA) using the method suggested by the manufacturer (Molecular Probes). Approximately $1 \times 10^{10} \mathrm{CFU}$ was harvested from a CBA plate, washed in PBS, and resuspended in $400 \mu \mathrm{L}$ of $5 \mu \mathrm{M}$ CellTracker Green CMFDA diluted in BHI media. The bacteria were incubated for $30 \mathrm{~min}$ at $37^{\circ} \mathrm{C}$, pelleted again by centrifugation, resuspended in $1 \mathrm{~mL}$ BHI media, and again incubated for $30 \mathrm{~min}$ at $37^{\circ} \mathrm{C}$. The bacteria were washed five times in Hank's buffered salt solution (HBSS) with $0.2 \%$ BSA and after the final wash their concentration was adjusted to $2 \times 10^{8} \mathrm{CFU} / \mathrm{mL}$ by estimation from the OD at $400 \mathrm{~nm}$. Twenty microliter of the bacterial suspension was mixed with $10 \mu \mathrm{L}$ of mouse serum and incubated for $30 \mathrm{~min}$ at $37^{\circ} \mathrm{C}$ with gentle shaking $(150 \mathrm{rpm})$. The serum samples were diluted to approximately the same relative antibody concentration as determined by ELISA (approximately $20 \mu \mathrm{g} / \mathrm{mL}$ ) so that the results would reflect the opsonizing ability of the antibodies rather than to differences associated with titer to the specific antigens.

Ten microliter of guinea pig serum was added as an exogenous complement source and the mixture was incubated for a further $15 \mathrm{~min}$ at $37^{\circ} \mathrm{C}$ with gentle shaking. Mouse macrophages (RAW 264.7) were washed twice with HBSS and once with HBSS containing $\mathrm{Ca}^{2+}$ and $\mathrm{Mg}^{2+}$ and the concentration of cells was adjusted to $2.5 \times 10^{6}$ cells $/ \mathrm{mL}$. Forty microliter of this macrophage suspension was added to the bacterial suspension. The mixture was incubated for $30 \mathrm{~min}$ at $37^{\circ} \mathrm{C}$ with gentle shaking to allow phagocytosis of the bacteria by the macrophages. The cells were diluted with $200 \mu \mathrm{L}$ of HBSS with $\mathrm{Ca}^{2+}$ and $\mathrm{Mg}^{2+}$ with $0.2 \% \mathrm{BSA}, 50 \mu \mathrm{L}$ of $0.5 \mathrm{mg} / \mathrm{mL}$ trypan blue was added to quench the fluorescence of extracellular bacteria and the cells were kept on ice before analysis using a flow cytometer (Beckman Coulter XL-MCL).

The relative fluorescence of the macrophages was used to assess the degree of phagocytosis in each sample. A higher percentage of fluorescent macrophages indicated a higher percentage of these cells had phagocytosed bacteria. Controls were included that received buffer instead of the mouse serum and serum from either non-immunized mice or mice immunized with whole killed $M$. catarrhalis 4223 (WKC) were included as negative and positive controls, respectively.

\section{MOUSE LUNG CHALLENGE MODEL WITH MUCOSAL IMMUNIZATION}

The mucosal immunization regime consisted of an intra-Peyer's patch (IPP) immunization followed 14 days later by an intratracheal (IT) boost, following the method developed by Kyd et al. (1999). Each mouse received $10 \mu \mathrm{g}$ of recombinant protein antigen or $10^{8} \mathrm{CFU}$ of formalin-killed bacteria in each immunization.
The mice were immunized with one of the following antigens: (1) whole killed M. catarrhalis 4223; (2) M35; (3) M35mat; (4) M35loop3 $3^{-}$; (5) L3 ${ }_{\text {DHFR }}$; and (6) DHFR. Five male BALB/c mice were included in each immunization group and a non-immunized control group was included in each experiment. Protein concentrations were determined with a micro BCA assay kit (Pierce) and adjusted to $2 \mathrm{mg} / \mathrm{mL}$. Proteins were concentrated using Amicon Ultra-15 Centrifugal Filter Units (Millipore) with a molecular weight limit of $5 \mathrm{kDa}$ and final adjustments were made by dilution with $10 \mathrm{mM}$ Tris-Cl, $\mathrm{pH}$ 7.4. As the M35-derived proteins precipitate without the presence of a denaturing agent or detergent, urea was added to final concentration of urea of $2.5 \mathrm{M}$.

Pulmonary challenge with $1 \times 10^{7}$ CFU live M. catarrhalis 4223 was carried out 21 days after the IPP immunization, following the protocol described by Kyd et al. (1999). This experiment was undertaken with the approval of the Central Queensland University Animal Ethics Committee.

\section{STATISTICAL ANALYSIS}

For the opsonophagocytosis assay triplicate values for the percentage of macrophages with fluorescence greater than the background for each sample were averaged and compared by one-way analysis of variance (ANOVA) with Tukey's multiple comparison test. Values for bacterial recovery and antibody concentrations for each immunization group were compared to the non-immunized control group using a one-way ANOVA followed by Bonferroni's multiple comparison test. Correlation of results was assessed using a Pearson's test. All analyses were performed using GraphPad Prism 4.0b.

\section{RESULTS}

\section{ANTIBODY-BINDING TO SURFACE EPITOPES OF M35}

Surface accessibility of the recombinant M35 proteins and loop 3 of M35 was investigated by determining the ability of antibody to bind to surface epitopes of M35 in M. catarrhalis isolates 4223 and ID78LN266 by flow cytometry (Figure 2). Antibodies raised against M35 bound to the cell surface of $M$. catarrhalis as demonstrated by a small right-shift in the relative fluorescence compared to the control non-immune serum. While this shift was small, it was reproducible and consistent with our previous results (Easton et al., 2005). Antibodies raised against M35(ID78) and M35loop3 ${ }^{-}$, respectively, caused similar sized right-shifts to that seen with anti-M35 serum. Antibodies raised against L3 ${ }_{\text {DHFR }}$ and DHFR caused smaller right-shifts than those seen with the anti-M35 serum. The lack of difference between the right-shifts seen with $\mathrm{L} 3_{\text {DHFR }}$ and DHFR antisera, indicates that any binding was not due to loop 3-specific recognition by the serum antibodies.

The binding of antibodies to M. catarrhalis differed between the strains of bacteria used. Antibodies raised in mice immunized with isolate 4223 demonstrated a greater ability to bind to whole killed cells of strain 4223 than that seen when tested against whole killed cells from strain ID78LN266. This may indicate that these two isolates differ substantially in the availability of outer membrane protein epitopes that are targeted by the antibodies. It was also noted that the background staining with naïve serum was higher 

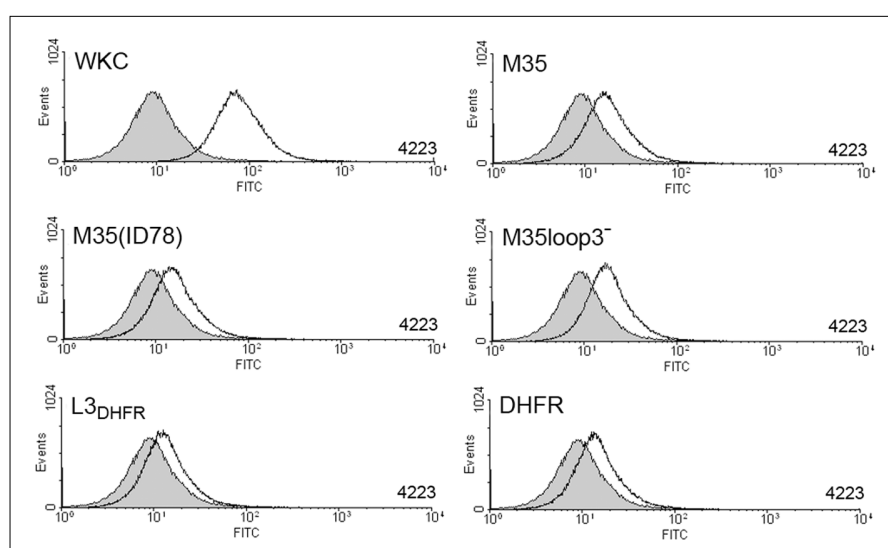

FIGURE 2 | Flow cytometry histograms showing the relative fluorescence of $M$. catarrhalis ID78LN266 incubated with antibodies raised against the $\mathbf{M} 35$-derived constructs. The strain is indicated in the lower right-hand corner of each panel and the antigen against

with isolate ID78LN266 than isolate 4223 (data not shown), suggesting that isolate ID78LN266 may express surface components with epitope sequences homologous to the normal flora of the mice. This difference between the strains may partly explain the lack of significant binding between the naïve sera and M35-specific antibodies to isolate ID78LN266 in our previous study (Easton et al., 2005).

\section{BACTERICIDAL ACTIVITY OF M35-SPECIFIC ANTIBODIES}

In an in vitro bactericidal assay, a greater than $50 \%$ killing of bacteria was achieved using anti-serum raised against the whole formalin-killed M. catarrhalis cells (WKC; Figure 3). None of the antisera raised against loop 3 or the M35-derived proteins were bactericidal (Figure 3).

\section{ENHANCEMENT OF OPSONOPHAGOCYTOSIS BY M35-SPECIFIC ANTIBODIES}

The ability of antibodies raised against each of the recombinant proteins to opsonize and therefore enhance phagocytosis of live M. catarrhalis cells was tested by determining the percentage of macrophages that had phagocytosed fluorescently labeled live $M$. catarrhalis. Using flow cytometric analysis the fluorescent intensity of the macrophages was assessed following incubation with fluorescently labeled bacteria in the presence or absence of antisera (Figure 4). Bacteria exposed to the 4223 WKC anti-serum as a positive control were readily phagocytosed with nearly $50 \%(48 \pm 1.3 \%)$ of macrophages showing greater than background fluorescence $(p<0.001)$. An average of $9.8 \pm 0.2 \%$ of macrophages phagocytosed bacteria that were exposed to serum from non-immunized mice and this was a similar percentage to the control sample exposed to sterile PBS instead of serum $(10.8 \pm 0.84 \%)$. Antibodies against L3 $3_{\mathrm{DHFR}}$ and DHFR both failed to enhance phagocytosis, with results of $11.8 \pm 0.6$ and $10.7 \pm 0.4 \%$, respectively. Antibodies raised against M35, M35(ID78), and M35loop3- all enhanced phagocytosis with a small significant increase in fluorescence of $15.6 \pm 0.8 \%$ $(p<0.05), 17.2 \pm 1.6 \%(p<0.01)$, and $16.4 \pm 2.3 \%(p<0.01)$, respectively (Figure 4 ).
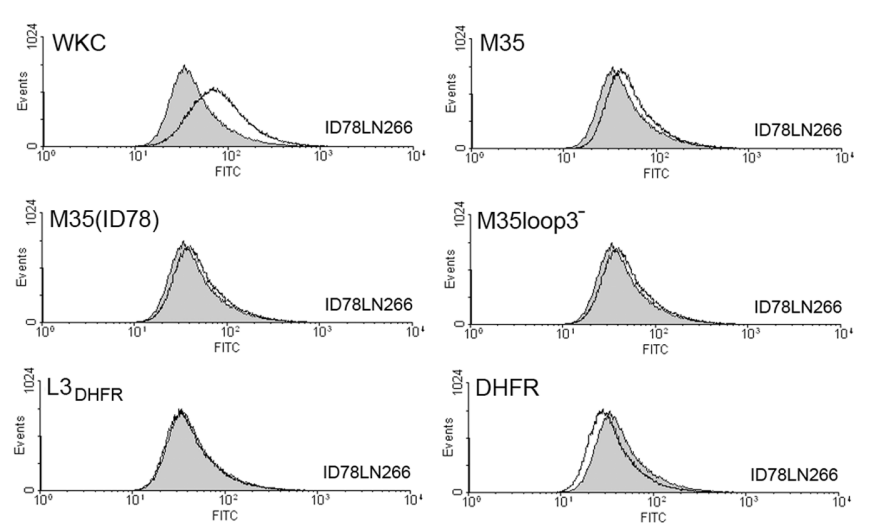

which the anti-serum was raised is indicated in the top left-hand corner of each panel. WKC; whole killed cell, formalin-killed

M. catarrhalis 4223. The gray histograms represent non-immune control serum.

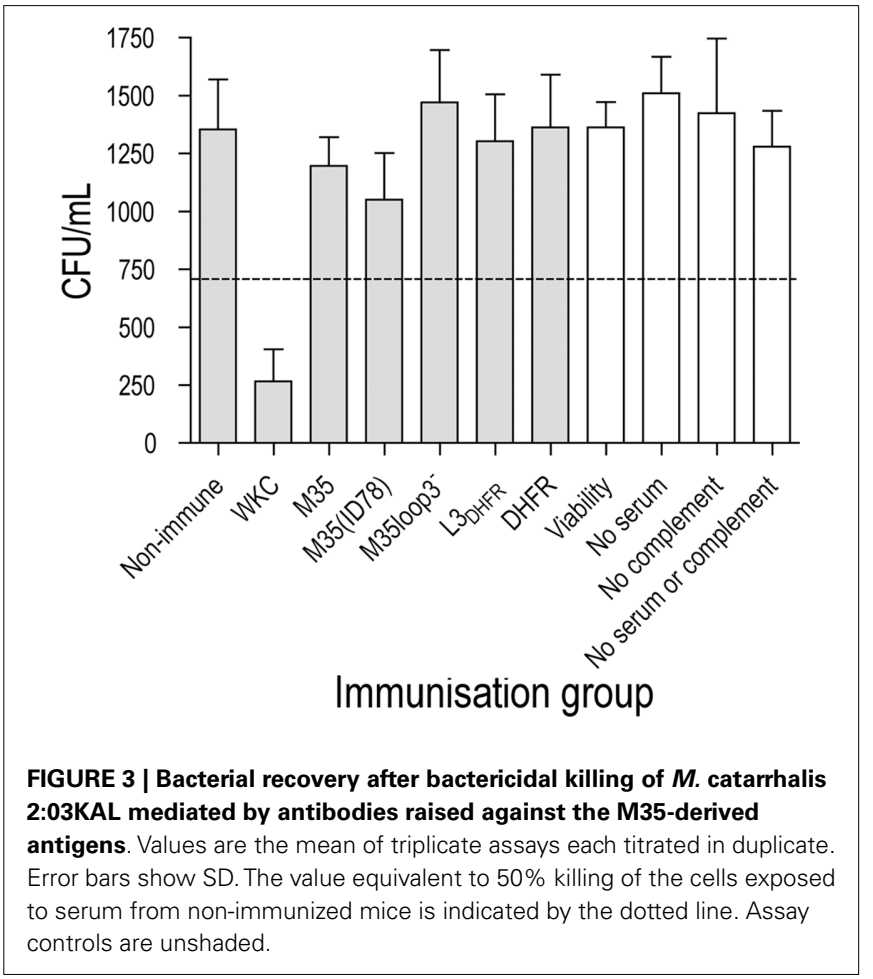

\section{BACTERIAL RECOVERY FROM MOUSE LUNGS AFTER CHALLENGE WITH LIVE M. CATARRHALIS}

The effect of mucosal immunization with each M35-derived construct was assessed by quantification of $M$. catarrhalis recovered from the lungs of mice $4 \mathrm{~h}$ after challenge by intra-tracheal inoculation with live bacteria. The quantity of live bacteria recovered from the BAL fluid, lung homogenate and in total from mice mucosally immunized with each antigen is shown in Figure 5. When compared to the non-immunized mice the number of bacteria recovered from mice mucosally immunized with the formalinkilled M. catarrhalis 4223 cells (WKC) were significantly lower in both the BAL $(p<0.001)$ and lung homogenate $(p<0.01)$. 


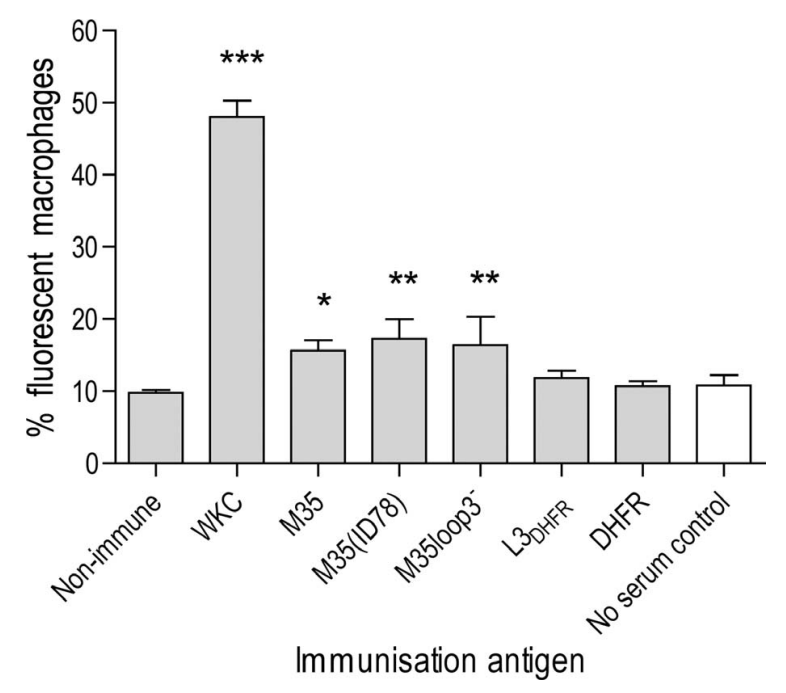

FIGURE 4 | Percentage of macrophages with enhanced fluorescence due to phagocytosis of $M$. catarrhalis 2:03KAL opsonized by antibodies raised against the M35-derived antigens. Values are the mean of triplicate assays. Error bars show SD. Asterisks indicate statistical significance compared to the non-immunized control group: ${ }^{*} p<0.05$, ${ }^{* *} p<0.01,{ }^{* * *} p<0.001$. The assay control is unshaded.

The bacterial recovery in the lung homogenate from mice mucosally immunized with M35, M35(ID78), and M35loop3- was also significantly lower than from the non-immunized mice $(p<0.01)$, however in the BAL fluid, enhancement of bacterial clearance only reached significance when M35(ID78) was the immunizing antigen $(p<0.01)$. When BAL and lung values were added to give total bacterial recovery (Figure 5A) all three groups [M35, M35(ID78), and M35loop3 ${ }^{-}$] had significantly lower numbers of bacteria recovered in total than in the non-immunized control group $(p<0.01)$. Bacterial recovery from the mice immunized with M35mat, L3 ${ }_{\text {DHFR }}$, and DHFR did not differ significantly from the non-immunized control groups in either the BAL fluid, lung homogenate, or total recovery $(p>0.05)$.

\section{ANTIBODY TITER AFTER MUCOSAL IMMUNIZATION}

IgG and IgA concentrations in the BAL fluid of immunized mice were very low and were undetectable in many samples (Table 1). The results from individual mice were found to be variable within the immunization groups, as indicated by the large SDs shown in Table 1. The concentration of IgG in the BAL tended to reflect the concentration in the serum for each individual animal. The nonimmunized mice did not have any detectable anti- $M$. catarrhalis antibody. Serum IgG antibody to loop 3 was very low when detected and was undetectable in most of the mice immunized with L3 $3_{\text {DHFR }}$ and none of those immunized with DHFR (Table 1).

\section{WHITE BLOOD CELL RECOVERY FROM MUCOSALLY IMMUNIZED MICE}

The recruitment of white blood cells to the lungs is associated with both innate and acquired immune responses to infection. In general, the total white blood cells recovered at $4 \mathrm{~h}$ post challenge is significantly higher in the WKC immunized groups when compared with the non-immune groups and this pattern was

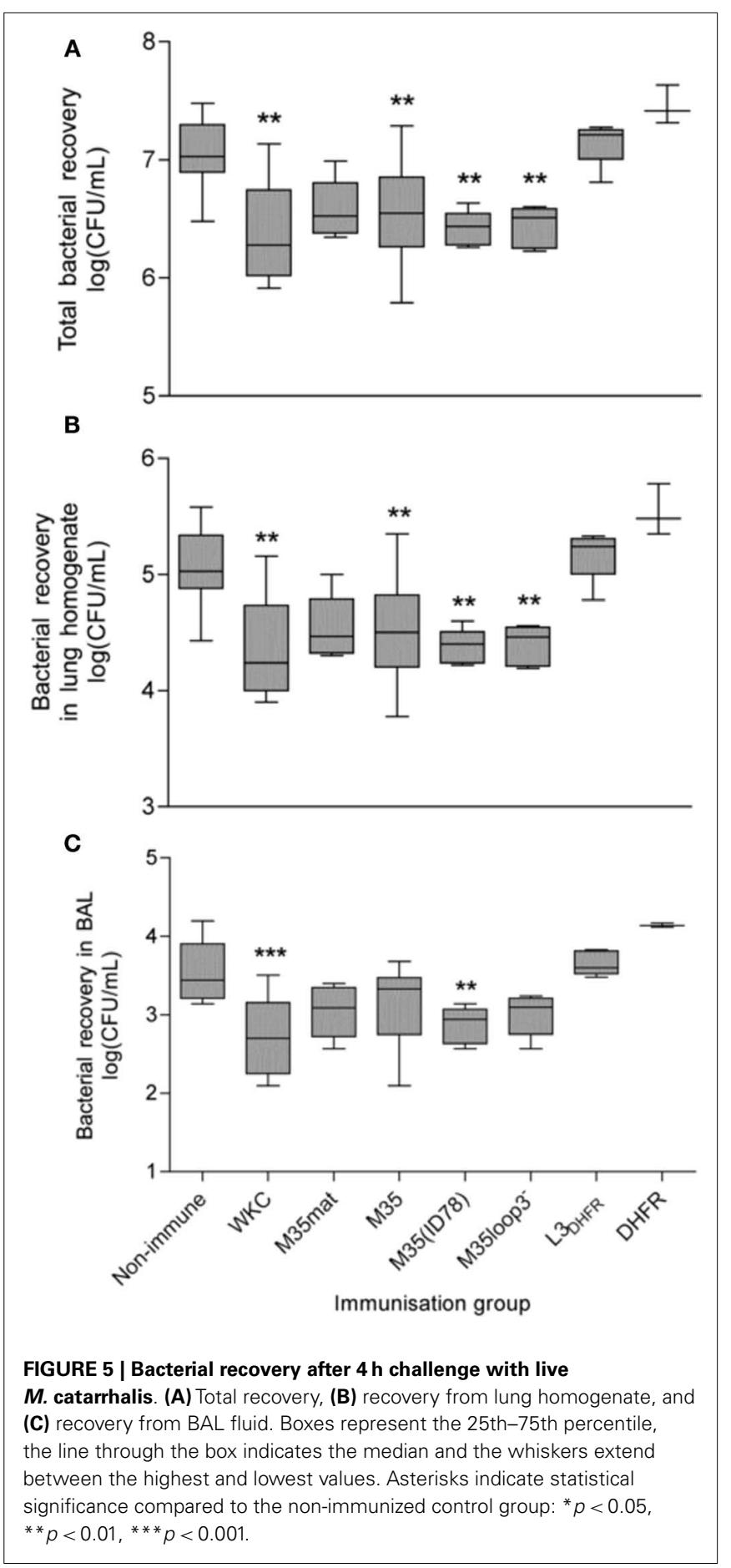

replicated in this study (Figure 6A). The total number of white blood cells recruited to the lungs and counted in the BAL was highest in the L3 ${ }_{\text {DHFR }}$ group ( $p<0.001$; Figure 6A). The most abundant cell type were neutrophils (Figure 6B) followed by monocytes/macrophages (Figure 6C) and in general the proportions followed the same trend as the total white blood cell counts for each group (Figure 6A), with the exception that there was significantly more monocyte/macrophage cells recovered from the WKC group compared to the non-immunized mice $(p<0.01)$. 
Table 1 | Specific antibody levels in serum and BAL after mucosal immunization.

\begin{tabular}{llll}
$\begin{array}{l}\text { Immunization } \\
\mathbf{g r o u p}^{\mathbf{a}}\end{array}$ & $\begin{array}{l}\text { Serum } \mathbf{I g G} \\
(\boldsymbol{\mu} \mathbf{g} / \mathbf{m L})^{\mathbf{b}}\end{array}$ & $\begin{array}{l}\text { BAL } \mathbf{g G G} \\
(\boldsymbol{\mu} \mathbf{g} / \mathbf{m L})\end{array}$ & $\begin{array}{l}\mathbf{B A L} \mathbf{g} \mathbf{A} \\
(\boldsymbol{\mu} \mathbf{g} / \mathbf{m L})\end{array}$ \\
\hline Non-immune & n.d. $^{\mathrm{c}}$ & n.d. & n.d. \\
WKC & $2.25 \pm 1.07$ & $0.34 \pm 0.34$ & n.d. \\
M35 & $16.4 \pm 25.6$ & $0.25 \pm 0.75$ & $0.01 \pm 0.04$ \\
M35mat & $54.4 \pm 42.6$ & $0.49 \pm 0.45$ & $0.01 \pm 0.03$ \\
M35(ID78) & $75.8 \pm 47.8$ & $1.23 \pm 0.95$ & $0.02 \pm 0.04$ \\
M35loop3 & $52.9 \pm 62.1$ & $0.60 \pm 0.65$ & n.d. \\
L3 & $0.064 \pm 0.14$ & n.d. & n.d. \\
DHFR & n.d. & n.d. & n.d.
\end{tabular}

aThe antibody concentration was measured for each mouse individually against the same antigen with which the mouse was immunized and the samples from the non-immunized mice were tested for antibody against both M35 and the whole $M$. catarrhalis cells. DHFR was assayed against $L 3_{\text {DHFR }}$.

${ }^{b}$ Values are mean and SD.

${ }^{c}$ Not detected.

\section{CORRELATION BETWEEN BACTERIAL CLEARANCE AND OPSONOPHAGOCYTOSIS}

To determine if there was a direct relationship between the ability of the animals to clear the infection challenge and the opsonophagocytosis results, a Pearson's test was conducted. There was a direct and significant correlation (Figure 7) between the total bacterial recovery from mice in each immunization group (Figure 5A) and the enhancement of opsonophagocytosis mediated by serum antibody raised against the same immunization antigens (Figure $4 ; p=0.001$ ).

\section{DISCUSSION}

M35 is an OMP from M. catarrhalis that we have been investigating for its functional role and potential as a vaccine antigen. Our initial characterization of M35 suggested that it possesses the desired characteristics of a vaccine antigen in that it is highly conserved, surface exposed, and immunogenic in an animal model (Easton et al., 2005). Our previous study also indicated that the loop 3 region of M35 was immunodominant over the remainder of the protein when mice were immunized with recombinant refolded M35 protein and raised the possibility that this loop may not be accessible to antibodies in the native protein expressed on the surface of the bacteria. Thus a situation may exist where the majority of antibodies produced against M35 are not effective due to an inability to bind to the protein on the cell surface. This would also be also consistent with previous observations relating to other porin proteins such as the loop 3 of the E. coli OMP F porin protein which has a major $\mathrm{B}$ cell region within the loop 3 domain (Williams et al., 2000; Yamashita et al., 2008). To investigate this hypothesis we have assessed the functional activity of antibodies specific to M35 and its loop 3 region.

This study has established that whilst at least some of the antibodies raised against the full M35 protein are able to bind to the protein on the cell surface, those specific for loop 3 do not bind a surface accessible epitope. The functional assays have demonstrated that while M35-specific antibodies were not bactericidal,

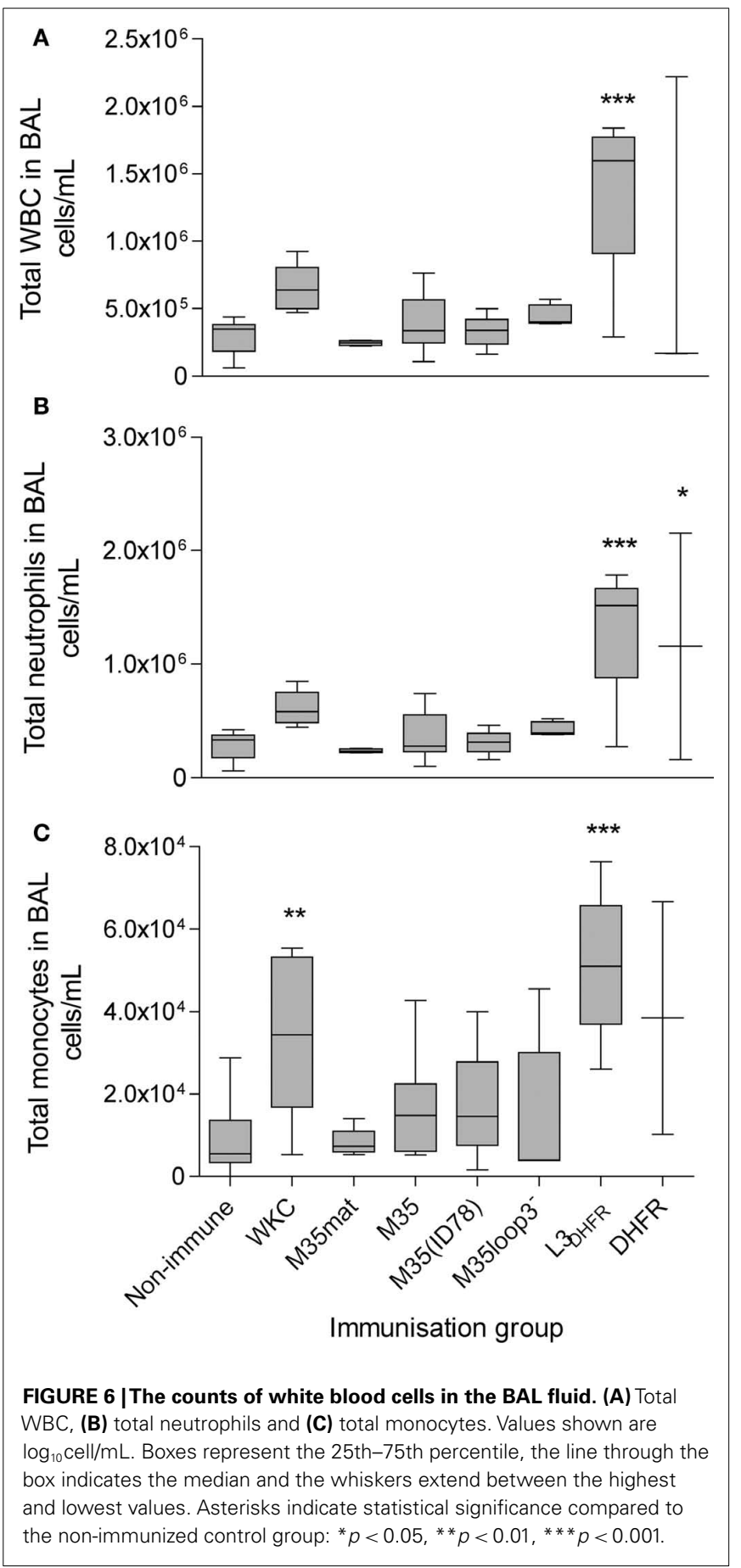

antibodies to intact M35 constructed proteins were opsonizing in vitro. Loop 3-specific antibodies were neither opsonizing nor bactericidal, providing further evidence that antibodies specific for this immunodominant epitope are unlikely to be effective against infection. While this is the longest of the external loop regions, the lack of surface recognition, or opsonizing activity by loop 3specific antibody supports the proposed structural model that this loop folds into the channel formed by the protein (Easton et al., 2005). Bactericidal activity has been used to assess functional activity for other M. catarrhalis antigens. Human antibodies to MID, 


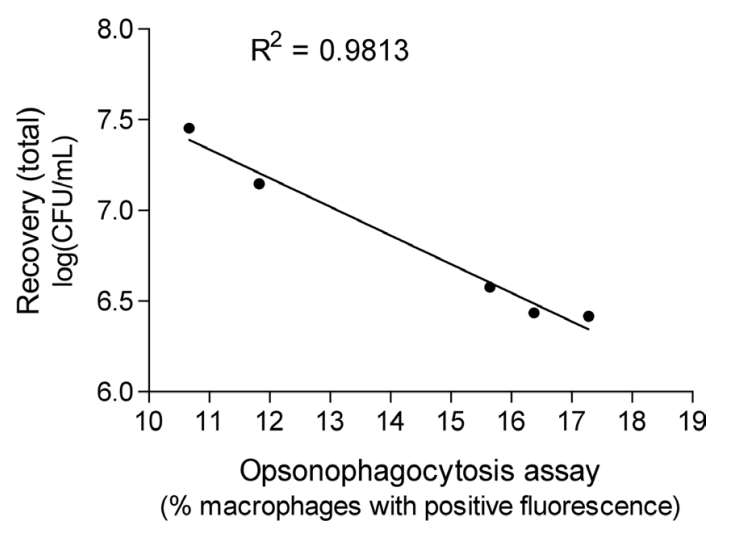

FIGURE 7 |The correlation between average total bacterial recovery after mucosal immunization and the enhancement of phagocytosis of $M$. catarrhalis due to opsonization by antibody raised against the same antigen.

UspA1, and UspA2 (Tan et al., 2006) and mouse antibodies dLOS, CD, and UspA (Hu et al., 2004) have been shown to be bacterical in vitro. Therefore in the current study, antibody to antigens other than M35 were responsible for the bacterical killing by WKC antisera.

The efficacy of potential vaccine antigens against $M$. catarrhalis has been modeled using the murine mucosal immunization pulmonary challenge model, in which effective immunization enhances the clearance of live bacteria from the lungs of mice (Murphy et al., 1998; Kyd et al., 1999). At present there is no correlate of protection for $M$. catarrhalis in this model and it is unknown whether antigens found to be effective in this model will also be effective in humans (Murphy, 2005, 2009). Attempts to determine the mechanism of protection in the mouse model have investigated the quantity, type and in vitro bactericidal capacity of antibodies produced by the mice, however none of these parameters has been shown to be definitive correlates of protection (Murphy et al., 1998; Kyd et al., 1999). For example, Murphy et al. (1998) found that while mucosal immunization led to the production of bactericidal antibody, intramuscular immunization did not, but both routes were similarly effective at enhancing clearance of the bacteria, suggesting that the bactericidal capacity of the antibodies was not necessarily a correlate of protection in an acute infection mode identical to that described in the current study. Differences between the naïve and immune-induced infiltration of phagocytic cells can be an indicator of antigen specific upregulation of the host's immune response to the infection. Therefore we hypothesized that immunization would enhance Fc-mediated phagocytic removal of live bacteria via enhanced opsonization. Sera from WKC immunized mice demonstrated the most significant opsonizing activity and these mice also showed the greatest level of enhanced bacterial clearance in the lung infection study. In contrast, the $\mathrm{L} 3_{\text {DHFR }}$ immunization was the least effective and the antisera was not opsonizing. Analysis of the correlation of the opsonic capacity of M35-specific antibodies with bacterial clearance in vivo showed a linear relationship, indicating a direct correlation between the two measures. This is an important finding in that it raises the question of whether opsonizing antibody would also be protective in humans, or whether it only appears to be protective in the mice due to the short time-frame of the challenge model and perhaps even just for this antigen. Also of interest is that there does not appear to be a relationship between the number cells recruited to the lung following challenge and clearance. However, if opsonizing antibodies are not induced a large cellular influx does not result in enhanced bacterial clearance from the lung. This is most striking for the $\mathrm{L} 3_{\text {DHFR }}$ data.

A hallmark of respiratory infections is the rapid recruitment of white cells to the bronchial space following infection. This recruitment into the lung is in response to bacterial lipopolysacharide and a range of inflammatory mediators that are released by resident phagocytes and endothelial cells of the bronchus (Dunkley et al., 1995). Immunization with antigens from NTHi, Streptococcus pneumoniae and Pseudomonas aeruginosa have previously been reported to enhance the recruitment of white cells (both neutrophils and monocytes) when the lung is challenged with live microbes (Buret et al., 1994; Foxwell et al., 2001; Jomaa et al., 2005b). Furthermore, this recruitment is antigen dependent with immunization by WKCs normally be the most effective (Jomaa et al., 2005b). However, in a previous study of M. catarrhalis, we did observe that immunization with purified OMP CD was as effective as WKC in enhancing the recruitment of white cells to the lung (Murphy et al., 1998). In the current study we observed that whilst WKC immunization was effective in enhancing the recruitment of monocytes to the lung, L 3 DHFR was actually more effective and enhanced the recruitment of neutrophils as well. The mechanism by which immunization is able to enhance the recruitment of white cells to the lung is most likely T cell dependent (Khader et al., 2009). It is thought that antigen specific T cells are generated in the gut associated lymphoid tissue following immunization and home to the lung via the common mucosal immune system (Otczyk and Cripps, 2010; Clancy and Dunkley, 2011) where they secrete a range of cytokines and chemokines that result in the recruitment of further neutrophils (Clancy and Dunkley, 2011). In this scenario a plausible explanation for the significantly enhanced recruitment of cells after immunization with L $3_{\text {DHFR }}$ would be the presence of T cell epitopes within the construct.

Although the role of opsonizing antibody has been speculated upon (Murphy et al., 2003), the only study demonstrating the development of oponizing antibody showed that antisera from 11 children with $M$. catarrhalis otitis media had increased opsonic activity in convalescent sera $(34.6 \pm 27.1)$ compared to acute sera (15.5 $\pm 6.7 ; p<0.05$; Faden et al., 1994). We are not aware of any other studies that have shown a correlation between antibody responses to a $M$. catarrhalis antigen and opsonic activity in the absence of bactericidal activity. It will be important to test in future studies whether this is specific to immunization with the M35-derived proteins or whether enhancement of in vitro phagocytosis is also correlated with protection after immunization with other M. catarrhalis antigens. A similar observation was reported for N. meningitidis, where the majority of PorB-specific IgG was not bactericidal but was strongly opsonic (Delvig et al., 1995, 1996, 1997b).

Anti-M35 serum demonstrated weak binding to M35loop3-, no binding to DHFR, weak binding to M35(ID78) but strong 


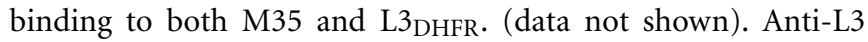
sera showed strong recognition of the M35 proteins, demonstrating that while there are conformational differences between the L3 regions of the whole M35 and the L3 3 DHFR construct, there is cross recognition of the epitopes in common between the peptide and the recombinant whole protein. Antibodies specific for M35, M35(ID78), and M35loop3 ${ }^{-}$were all able to bind to the surface of the bacterium, meaning that the presence, absence, or alteration of loop 3 did not affect the recognition of surface epitopes of M35. However, loop 3-specific antibodies were unable to bind to M35 on the surface of M. catarrhalis, supporting the prediction that the loop 3 region would not be accessible to antibodies, as it was predicted that it folded into the porin channel (Easton et al., 2005). Analysis of the in vitro opsonophagocytic activity of the M35-specific antibodies revealed that antibodies specific for the loop 3 region were not able to enhance phagocytosis, presumably due to their inability to bind on the cell surface. Antibodies raised against M35, M35(ID78), and M35loop3- ${ }^{-}$were all able to enhance in vitro phagocytosis, demonstrating that it was antibody directed at the surface region that was responsible for the increase in phagocytosis and also that deletion of loop 3 did not create alterations to the protein that affected production of antibody with opsonizing activity. It therefore appears that, unlike the N. meningitidis PorB where the immunodominant region was in loop 1 (Delvig et al., 1995, 1996, 1997b) and surface exposed, the majority of antibodies raised against $M$. catarrhalis M35 are against loop 3 and are not opsonizing due to specificity for an apparently hidden loop region.

Preliminary studies with the native protein had demonstrated the efficacy of M35 as a protective antigen when used in mucosally immunized mice prior to pulmonary challenge with live $M$. catarrhalis (unpublished data). Since our subsequent investigations had suggested that the majority of antibodies produced following immunization were directed at loop 3, it was hypothesized that immunization with loop 3 alone would not be protective. Mice immunized with M35, M35(ID78), and M35loop3 ${ }^{-}$cleared significantly more of the bacteria from their lungs than the nonimmunized control mice, indicating that deletion of the loop 3 region from the protein did not reduce, or enhance, the efficacy of the M35-specific response to infection. Mice mucosally immunized with the loop 3 peptide were not able to enhance clearance of the bacteria, however, these mice produced very low levels of L3 ${ }_{\text {DHFR }}$-specific IgG detectable only in the sera and no IgG or IgA was detected in the BAL. However, mice immunized with M35 that contains an antigenically different loop 3 M35(ID78) (Easton

\section{REFERENCES}

Aase, A., Bjune, G., Hoiby, E. A., Rosenquist, E., Pedersen, A. K., and Michaelsen, T. E. (1995). Comparison among opsonic activity, antimeningococcal immunoglobulin $\mathrm{G}$ response, and serum bactericidal activity against meningococci in sera from vaccines after immunization with a serogroup B outer membrane vesicle vaccine. Infect. Immun. 63, 3531-3536.
Armbruster, C. E., Hong, W., Pang, B., Weimer, K. E., Juneau, R. A., Turner, J., and Swords, W. E. (2010). Indirect pathogenicity of Haemophilus influenzae and Moraxella catarrhalis in polymicrobial otitis media occurs via interspecies quorum signaling. MBio 1, e00102-e00110.

Bash, M. C., Lesiak, K. B., Banks, S. D., and Frasch, C. E. (1995). Analysis of Neisseria meningitidis class 3 outer membrane protein gene

et al., 2005) or had the loop 3 region deleted (M35loop3 ${ }^{-}$) had similar immune efficacy against infection, indicating that antibody generated against loop 3 from whole protein immunization was not involved in M35-specific bacterial clearance. Therefore, while clearance of $M$. catarrhalis in the acute mouse lung challenge model could be enhanced by mucosal immunization with M35, this was independent of loop 3 as predicted. Studies with NTHi OMP P5 found that the fourth surface exposed region of the P5 antigen from NTHi was immunodominant over the remainder of the protein and not protective, however, immunization with the third surface exposed region of P5 was protective (Novotny and Bakaletz, 2003). It is possible that a similar pattern could exist with M35, suggesting further investigation of the antigenic roles of specific surface loops of M35 is warranted.

\section{CONCLUSION}

This study has confirmed our prediction that the majority of antibodies raised against M35 that were specific for loop 3 are ineffective and unlikely to be associated with immune protection against infection. The study has found that there was a correlation between opsonizing antibodies associated with enhanced phagocytosis and in vivo enhancement of bacterial clearance in mucosally immunized mice. Immunization with M35 with or without loop 3 was effective in enhancing clearance in the mouse pulmonary challenge model, suggesting that the enhancement of opsonophagocytosis due to antibodies to other surface epitopes contributed to this response. The study suggests that opsonic antibodies are potentially important for enhanced clearance and the role of opsonic antibody to $M$. catarrhalis should be further investigated for other antigens.

\section{AUTHORIZATION FOR THE USE OF EXPERIMENTAL ANIMALS}

Animal studies were undertaken with the approval of the Central Queensland University Animal Ethics Committee and the University of Canberra Committee for Ethics in Animal Experimentation.

\section{ACKNOWLEDGMENTS}

We wish to acknowledge the assistance of John McGrath, Ajay Krishnamurthy, Jessica Browne, Claire Batum, and Nancy Fisher with the immunization experiments. The authors also wish to thank the reviewers for their engagement with the data presented in this paper and their very helpful suggestions which improved both the presentation and interpretation of the findings.

variable regions and type identification using genetic techniques. Infect. Immun. 63, 1484-1490.

Buret, A., Dunkley, M. L., Pang, G., Clancy, R. L., and Cripps, A. W. (1994). Pulmonary immunity to Pseudomonas aeruginosa in intestinally immunized rats roles of alveolar macrophages, tumor necrosis factor alpha, and interleukin-1 alpha. Infect. Immun. 62 5335-5343.
Clancy, R., and Dunkley, M. (2011). Acute exacerbations in COPD and their control with oral immunization with non-typeable Haemophilus influenzae. Front. Immun. 2:7. doi: 10.3389/fimmu.2011.00007

Daoud, A., Abuekteish, F., and Masaadeh, H. (1996). Neonatal meningitis due to Moraxella catarrhalis and review of the literature. Ann. Trop. Paediatr. 16, 199-201. 
Delvig, A. A., Michaelsen, T. E., Aase, A., Hoiby, E. A., and Rosenqvist, E. (1997a). Vaccine-induced IgG antibodies to the linear epitope on the PorB outer membrane protein promote opsonophagocytosis of Neisseria meningitidis by human neutrophils. Clin. Immunol. Immunopathol. 84, 27-35.

Delvig, A. A., Rosenqvist, E., Oftung, F., and Robinson, J. H. (1997b). T-Cell epitope mapping the PorB protein of serogroup B Neisseria meningitidis in B10 congenic strains of mice. Clin. Immunol. Immunopathol. 85, 134-142.

Delvig, A. A., Wedege, E., Caugant, D. A., Dalseg, R., Kolberg, J., Achtman, M., and Rosenquist, E. (1995). A linear B-cell epitope on the class 3 outer-membrane protein of Neisseria meningitidis recognized after vaccination with the Norwegian group B outer-membrane vesicle vaccine. Microbiology 141(Pt 7), 1593-1600.

Delvig, A. A., Wedege, E., Michaelsen, T. E., Hoiby, E. A., Brandtzaeg, P., and Rosenqvist, E. (1996). Immune responses to linear epitopes on the PorB protein of Neisseria meningitidis in patients with systemic meningococcal disease. Microbiology 142(Pt 9), 2491-2498.

Duim, B., Dankert, J., Jansen, H. M., and van Alphen, L. (1993). Genetic analysis of the diversity in outer membrane protein P2 of non-encapsulated Haemophilus influenzae. Microb. Pathog. 14, 451-462.

Dunkley, M., Pabst, R., and Cripps, A. (1995). An important role for intestinally derived $\mathrm{T}$ cells in respiratory defence. Immunol. Today 16, 231-236.

Easton, D. M., Smith, A., Gallego, S. G., Foxwell, A. R., Cripps, A. W., and Kyd, J. M. (2005). Characterization of a novel porin protein from Moraxella catarrhalis and identification of an immunodominant surface loop. J. Bacteriol. 187, 6528-6535.

Faden, H., Hong, J. J., and Pahade, N. (1994). Immune response to Moraxella catarhalis in children with otitis media: opsonophagocytosis with antigen-coated latex beads. Ann. Otol. Rhinol. Laryngol. 103, 522-524.

Foxwell, A. R., Kyd, J. M., and Cripps, A. W. (2001). Programmed inflammatory processes induced by mucosal immunisation. Vaccine 19, 2522-2526.

Haase, E. M., Campagnari, A. A., Sarwar, J., Shero, M., Wirth, M., Cumming,
C. U., and Murphy, T. F. (1991) Strain-specific and immunodominant surface epitopes of the $\mathrm{P} 2$ porin protein of nontypeable Haemophilus influenzae. Infect. Immun. 59, 1278 1284.

Hol, C., Verduin, C. M., Van Dijke, E. E., Verhoef, J., Fleer, A., and van Dijk, H. (1995). Complement resistance is a virulence factor of Branhamella (Moraxella) catarrhalis. FEMS Immunol. Med. Microbiol. 11, 207-211.

Hu, W. G., Berry, J., Chen, J., and Gu, X. X. (2004). Exploration of Moraxella catarrhalis outer membrane proteins, $\mathrm{CD}$ and UspA, as new carriers for lipooligosaccharidebased conjugates. FEMS Immunol. Med. Microbiol. 41, 109-115.

Jetter, M., Heiniger, N., Spaniol, V., Troller, R., Schaller, A., and Aebi, C. (2009). Outer membrane porin M35 of Moraxella catarrhalis mediates susceptibility to aminopenicillins. BMC Microbiol. 9, 188. doi: 10.1186/471-2180-9-188

Jomaa, M., Yuste, J., Paton, J. C. Jones, C., Dougan, G., and Brown, J. S. (2005a). Antibodies to the iron uptake ABC transporter lipoproteins PiaA and PiuA promote opsonophagocytosis of Streptococcus pneumoniae. Infect. Immun. 73, 6852-6859.

Jomaa, M., Kyd, J. M., and Cripps, A. W. (2005b). Mucosal immunisation with novel Streptococcus pneumoniae protein antigens enhances bacterial clearance in an acute mouse lung infection model. FEMS Immunol. Med. Microbiol. 44, 59-67.

Karalus, R., and Campagnari, A. (2000). Moraxella catarrhalis: a review of an important human mucosal pathogen. Microbes Infect. 2, 547-559.

Khader, S. A., Gaffen, S. L., and Kolls, J. K. (2009). Th17 cells at the crossroads of innate and adaptive immunity against infectious diseases at the mucosa. Mucosal Immunol. 2, 403-411.

Krishnamurthy, A., McGrath, J., Cripps, A. W., and Kyd, J. M. (2009). The incidence of Streptococcus pneumoniae otitis media is affected by the polymicrobial environment particularly Moraxella catarrhalis in a mouse nasal colonisation model. Microbes Infect. 11 545-553.

Kyd, J., John, A., Cripps, A., and Murphy, T. F. (1999). Investigation of mucosal immunisation in pulmonary clearance of Moraxella (Branhamella) catarrhalis. Vaccine 18, 398-406.
Kyd, J. M., Cripps, A. W., and Murphy, T. F. (1998). Outer-membrane antigen expression by Moraxella (Branhamella) catarrhalis influences pulmonary clearance. J. Med. Microbiol. 47, 159-168.

Martinez, J. E., Romero-Steiner, S. Pilishvili, T., Barnard, S., Schinsky, J., Goldblatt, D., and Carlone, G. M. (1999). A flow cytometric opsonophagocytic assay for measurement of functional antibodies elicited after vaccination with the 23-valent pneumococcal polysaccharide vaccine. Clin. Diagn. Lab. Immunol. 6 , 581-586.

Massa, H. M., Cripps, A. W., and Lehmann, D. (2009). Otitis media: viruses, bacteria, biofilms and vaccines. Med. J. Aust. 191, S44-S49.

Mawas, F., Ho, M. M., and Corbel, M. J. (2009). Current progress with Moraxella catarrhalis antigens as vaccine candidates. Expert Rev. Vaccines $8,77-90$.

Mes, T. H., and van Putten, J. P. (2007). Positively selected codons in immune-exposed loops of the vaccine candidate OMP-P1 of Haemophilus influenzae. J. Mol. Evol. 64, 411-422.

Meyer, G. A., Shope, T. R., Waecker, N. J. Jr., and Lanningham, F. H. (1995). Moraxella (Branhamella) catarrhalis bacteremia in children. A report of two patients and review of the literature. Clin. Pediatr. (Phila) 34 146-150.

Murphy, T. F. (1996). Branhamella catarrhalis: epidemiology, surface antigenic structure, and immune response. Microbiol. Rev. 60 267-279.

Murphy, T. F. (2005). Vaccine development for non-typeable Haemophilus influenzae and Moraxella catarrhalis: progress and challenges. Expert Rev. Vaccines 4, 843-853.

Murphy, T. F. (2009). Vaccine development for Moraxella catarrhalis: rationale, approaches and challenges. Expert Rev. Vaccines 8, 655-658.

Murphy, T. F., Kirkham, C., Liu, D. F., and Sethi, S. (2003). Human immune response to outer membrane protein $\mathrm{CD}$ of Moraxella catarrhalis in adults with chronic obstructive pulmonary disease. Infect. Immun. 71, 1288-1294.

Murphy, T. F., Kyd, J. M., John, A., Kirkham, C., and Cripps, A. W. (1998). Enhancement of pulmonary clearance of Moraxella (Branhamella) catarrhalis following immunization with outer membrane protein $\mathrm{CD}$ in a mouse model. J. Infect. Dis. 178 1667-1675.

Novotny, L. A., and Bakaletz, L. O. (2003). The fourth surface-exposed region of the outer membrane protein P5-homologous adhesin of nontypable Haemophilus influenzae is an immunodominant but nonprotective decoying epitope. J. Immunol. 171, 1978-1983.

Otczyk, D. C., and Cripps, A. W. (2010). Mucosal immunization: A realistic alternative. Hum. Vaccin. 6, 9781006.

Schirmer, T. (1998). General and specific porins from bacterial outer membranes. J. Struct. Biol. 121, 101-109.

Seshadri, K., Garemyr, R., Wallin, E., von Heijne, G., and Elofsson, A. (1998). Architecture of betabarrel membrane proteins: analysis of trimeric porins. Protein Sci. 7 2026-2032.

Tan, T. T., Christensen, J. J., Dziegiel, M. H., Forsgren, A., and Riesbeck, K. (2006). Comparison of the serological responses to Moraxella catarrhalis immunoglobulin D-binding outer membrane protein and the ubiquitous surface proteins A1 and A2. Infect. Immun. 74, 6377-6386

Verduin, C. M., Hol, C., Fleer, A. van Dijk, H., and van Belkum, A. (2002). Moraxella catarrhalis: from emerging to established pathogen. Clin. Microbiol. Rev. 15, 125-144.

Verduin, C. M., Hol, C., Van Dijke, E. Faber, J. A., Jansze, M., Verhoef, J., and Van Dijk, H. (1995). Assessment of complement-mediated killing of Moraxella (Branhamella) catarrhalis isolates by a simple method. Clin. Diagn. Lab. Immunol. 2, 365-368.

Watanabe, Y. (2002). Characterization of the refolding and reassembly of an integral membrane protein OmpF porin by low-angle laser light scattering photometry coupled with high-performance gel chromatography. J. Chromatogr. A 961 , 137-146.

Williams, K. M., Bigley, E. C. III, and Raybourne, R. B. (2000). Identification of murine B-cell and T-cell epitopes of Escherichia coli outer membrane protein $F$ with synthetic polypeptides. Infect. Immun. 68, 2535-2545

Yamashita, E., Zhalnina, M. V., Zakharov, S. D., Sharma, O., and Cramer, W. A. (2008). Crystal structures of the OmpF porin: function in a colicin translocon. EMBO J. 27, 2171-2180 
Yi, K., and Murphy, T. F. (1997). Importance of an immunodominant surface-exposed loop on outer membrane protein P2 of nontypeable Haemophilus influenzae. Infect. Immun. 65, 150-155.

Conflict of Interest Statement: The authors declare that the research was conducted in the absence of any commercial or financial relationships that could be construed as a potential conflict of interest. The porin protein known as M35 has been patented for vaccine use with the authors as named inventors.

Received: 14 January 2011; accepted: 21 April 2011; published online: 16 May 2011.
Citation: Easton DM, Cripps AW, Foxwell AR and Kyd JM (2011) Mucosal immunization with the Moraxella catarrhalis porin M35 induces enhanced bacterial clearance from the lung: a possible role for opsonophagocytosis. Front. Immun. 2:13. doi: 10.3389/fimmu.2011.00013

This article was submitted to Frontiers in Mucosal Immunity, a specialty of Frontiers in Immunology.
Copyright $(2011$ Easton, Cripps, Foxwell and Kyd. This is an open-access article subject to a non-exclusive license between the authors and Frontiers Media $S A$, which permits use, distribution and reproduction in other forums, provided the original authors and source are credited and other Frontiers conditions are complied with. 